\title{
Atopic dermatitis and diet
}

\author{
AP Oranje, * FB de Waard-van der Spek \\ Department of Dermato-Venereology, University Hospital Rotterdam, Rotterdam, the Netherlands. ${ }^{\star}$ Corresponding author, University Hospital \\ Rotterdam and Erasmus University, Dr Molewaterplein 60, 3015 GJ Rotterdam, The Netherlands, tel. +31 104636656 ; fax +31 10 4636707; \\ E-mail:aoranje@inter.nl.netorOranje@alkg.azr.nl
}

Verma KK, Khaitan BK, Ramam M. Childhood atopic dermatitis treated with specific food elimination.

JEADV 2000; 14: 520-521

Atopic dermatitis (AD) is a multifactorial genetic disease in which many known and even unknown factors play a part. The role of diet in the cause and treatment of AD is very controversial: several investigators believe that foods play a major part in young children with $\mathrm{AD}$, whereas others state that food allergy and intolerance occur in patients with $\mathrm{AD}$, but have no influence on eczema. We believe that diet has a place in the therapy of young children with AD. Adverse reactions to foods may influence the severity of $\mathrm{AD}$ in children younger than 5 years; in older children reactions may occur, but hardly have any effect on $\mathrm{AD}$. Food additives rarely play a part in exacerbating $\mathrm{AD}$. If a child reacts adversely to foods, $\mathrm{AD}$ does not necessarily improve when the specific food is avoided. In contrast, if sensitivity to a certain food has been proved, improvement of $\mathrm{AD}$ is not necessarily because of dietary restriction.

Allergic reactions to foods in young children with AD may be immediate (direct), late-onset immediate, and delayed. Immunological contact urticaria is a common manifestation of food allergy in young children with AD. The exact frequency of contact urticaria in $\mathrm{AD}$ is unknown, but in our opinion it is common in young children with $\mathrm{AD}$. In delayed-type reactions it is very difficult to identify food triggers from patient history. Dermatological features of food reactions include: urticaria, contact urticaria, exanthem and eczematous eruption. There are two recognized pathways in food allergy. First, foods penetrate the skin and reach mast cells (contact urticaria) or reach pro-inflammatory cells. Secondly, ingested food antigens rapidly cross the gastrointestinal barrier and reach proinflammatory cells in the skin. Non-dermatological reactions include vomiting, diarrhoea, abdominal pain and other less common symptoms such as rhinitis and asthma. ${ }^{1}$ The most severe reaction is anaphylaxis and occurs most frequently in older children. ${ }^{1}$ However, when atopic disease manifests in the skin, most reactions are skin related. ${ }^{2}$ About $40 \%$ of food-sensitized children with $\mathrm{AD}$ on a selective diet show significant improvement of eczema. ${ }^{2}$ Resano et al..$^{3}$ obtained greater than $40 \%$ good results in 74 children with $\mathrm{AD}, 64 \%$ of whom were sensitized to food allergens. In a 3-year follow-up study, a diet avoiding causal foods combined with adequate symptomatic therapy, led to an important remission in skin manifestations in these food-sensitized children. ${ }^{3}$ However, in a double-blind controlled cross-over trial involving 53 children with $\mathrm{AD}$, on an egg and cow's milk exclusion diet, Neild et al. reported positive results in only 10 of the children. This response rate to the diet was not statistically significant. ${ }^{4}$ Response to the diet was assessed in terms of areas affected, daytime and night-time itch, and topical steroid usage.

In refractory widespread $\mathrm{AD}$, a strict antigen avoidance regimen may be associated with improvement of $\mathrm{AD}$ where conventional treatments have failed. ${ }^{5}$ Devlin et al..$^{5}$ concluded this from a study in a series of 37 children with $\mathrm{AD}$, treated with an elementary diet under controlled conditions. Improvement in eczema was seen in $73 \%$ of the patients. There were no clinical or laboratory findings that could be used to predict the outcome. Drawbacks to the regimen were prolonged hospitalization, a fall in body weight and serum albumin concentration and a risk of anaphylactic shock.

Preventive effects of diets is another controversial issue. Some high-risk infants may benefit from maternal diet during lactation, but there is no documented beneficial effect of maternal diet during pregnancy. Convincing conclusions from long-term prospective studies on preventive effects of diets in young children are lacking or limited.

Very strict diets without consulting a dietician may lead to nutritional deficiencies. Nutritional deficiencies such as calcium and iron deficiency may develop, leading to growth retardation and even rickets. Therefore, avoidance of multiple foods is potentially hazardous and requires continued paediatric and dietary supervision. ${ }^{6}$ Anaphylaxis is a definite hazard of elimination diets. Practical precautions are difficult because of the unpredictability of violent reactions and uncertainty on the life-saving efficacy of injected adrenaline. ${ }^{7}$ Parents should be warned of this risk.

One problem in evaluating diets is that completely reliable food tests are lacking. The best information can be obtained by performing skin tests, such as the skin application food test (SAFT) in children younger than 4 years with $\mathrm{AD}$, and 
skin prick-prick test with fresh foods as specified by Dreborg. ${ }^{8}$ The SAFT is a reliable and child-friendly skin test for evaluating (suspected) food allergy in children younger than 4 years with AD. ${ }^{9}$ The skin prick test is the test of first choice for investigating immediate IgE-mediated reaction. The SAFT has been developed on the basis of the mechanism of contact urticaria. The SAFT and the skin prick-prick test correlate well. Significantly more positive results are obtained in serology, the radioallergosorbent test (RAST) for food allergens, than in the SAFT. Advice and strategy only based on serology by RAST has very limited value and leads to overtreatment. De Waard-van der Spek et al. observed a very good correlation between the SAFT and the oral challenge $(\kappa=0.86) .{ }^{9}$

Another problem is that many reported studies have shortcomings in terms of evaluating the severity of $\mathrm{AD}$ and the standardization of such systems. Observation and interpretation of the results of therapeutic or preventive interventions are important and at best can be performed by using standardized scoring systems, such as objective SCORAD (Scoring Atopic Dermatitis). ${ }^{10}$ We also developed a simpler scoring system called the 'three item severity' score for routine use. 11

Finally, the double-blind placebo-controlled oral challenge is the gold standard, but the assessment of positive or negative reactions is not well established and still a problem.

In conclusion, elimination diets and preventive diets are beneficial in a small percentage of young children with AD. In our opinion, food allergy plays a part in $20 \%$ of the children younger than 4 years with AD. A direct effect on eczema is observed in about four of 10 children with $\mathrm{AD}$ and proven food allergy.

\section{Acknowledgements}

The authors are indebted to Bhupendra Tank, Department of
Dermato-Venereology, Erasmus University Rotterdam, for a critial reading and correcting the English of this text.

\section{References}

1 Rance F, Kanny G, Dutau G, Moneret-Vautrin DA. Food hypersensitivity in children: clinical aspects and distribution of allergens. Pediatr Allergy Immunol 1999; 10: 33-38.

2 Oranje AP, Aarsen RS, Mulder PG, et al. Food immediate-contact hypersensitivity (FICH) and elimination diet in young children with atopic dermatitis. Preliminary results in 107 children. Acta Derm Venereol Suppl 1992; 176: 41-44.

3 Resano A, Crespo E, Fernandez Benitez M, et al. Atopic dermatitis and food allergy. J Invest Allergol Clin Immunol 1998; 8(5): 271-276.

4 Neild VS, Marsden RA, Bailes JA, Bland JM. Egg and milk exclusion diets in atopic eczema. Br J Dermatol 1986; 114: 117-123.

5 Devlin J, David TJ, Stanton RH. Elemental diet for refractory atopic eczema. Arch Dis Child 1991; 66: 93-99.

6 David TJ, Waddington E, Stanton RH. Nutritional hazards of elimination diets in children with atopic eczema. Arch Dis Child 1984; 59(4): 323-325.

7 David TJ. Anaphylactic shock during elimination diets for severe atopic eczema. Arch Dis Child 1984; 59(10): 983-986.

8 Dreborg S. Skin tests used in type 1 allergy testing. Position paper. Allergy 1989; 44(Suppl. 19): 1-59.

9 de Waard-van der Spek FB, Elst EF, Mulder PG, et al. Diagnostic tests in children with atopic dermatitis and food allergy. Allergy 1998; 53(11): 1087-1091.

10 Kunz B, Oranje AP, Labreze L, et al. Clinical validation and guidelines for the SCORAD index: consensus report of the European Task Force on Atopic Dermatitis. Dermatology 1997; 195: 10-19.

11 Wolkerstorfer A, de Waard van der Spek FB, Glazenburg EJ, et al. Scoring the severity of atopic dermatitis: three item severity score as a rough system for daily practice and as a pre-screening tool for studies. Acta Derm Venereol 1999; 79(5): 356-359.

\section{Visit the EADV website at: www.eadv.org}

